\title{
Rol de los marcadores ecográficos secundarios de primer trimestre para la detección de trisomía 21
}

\author{
Patricia Pérez-Moneo P. ${ }^{1}$, Belén Aparicio $N^{1}$, Taqua Blanca $R^{1}{ }^{1}$, Paloma Badía A. ${ }^{1}$, \\ Cristina García M. ${ }^{1}$, Reyes Balanzá C. ${ }^{1}$ \\ ${ }^{1}$ Servicio de Ginecología y Obstetricia, Hospital Universitario Dr. Peset, Valencia. España.
}

\section{RESUMEN}

Objetivo: Analizar los resultados de los marcadores ecográficos secundarios (hueso nasal, onda a del ductus venoso y regurgitación tricuspídea) y valorar su efectividad para la detección de trisomía 21 y su utilidad para la reducción del número de pruebas invasivas. Métodos: Tras la realización del test combinado de primer trimestre a toda paciente con un riesgo entre 1/101-1/1000 se realizó la valoración de los marcadores secundarios. Resultados: Desde Enero de 2014 a Mayo de 2015 se realizaron 2.660 test combinados del primer trimestre valorándose la edad materna, la traslucencia nucal y la PAPP-A y BhCG, teniendo una sensibilidad del $90 \%$ y una tasa de falsos positivos del 3,2\%. Hubo 10 fetos con trisomía 21. La sensibilidad de hueso nasal, ductus venoso y regurgitación tricuspídea fue del $22,2 \%, 50 \%$ y $50 \%$ y la especificidad del $99,8 \%, 96,9 \%$ y $98,8 \%$ respectivamente. La sensibilidad global del test contingente fue del $90 \%$, con una reducción de la tasa de falsos positivos al 1,6\%, lo que se reduciría de 171 a 148 el número de amniocentesis. Conclusión: El test contingente es una buena herramienta para reducir la tasa de falsos positivos respecto al test combinado sin disminuir la tasa de detección y con ello reducir la tasa de pruebas invasivas.

\section{PALABRAS CLAVE: Trisomia 21, test contingente, marcadores ecográficos secundarios, hueso nasal, ductus venoso, regurgitación tricuspídea}

\section{SUMMARY}

Aims: To analyze the results of the secondary sonographic markers (nasal bone, wave ductus venosus and tricuspid regurgitation) and evaluate its effectiveness for the detection of trisomy 21 and thus reduce the number of invasive tests. Methods: After completing the first trimester combined test, all patients with a risk between 1/101-1/1000 were evaluated the secondary sonographic markers. Results: From January 2014 to May 20152660 combined test being assessed maternal age, nuchal translucency and PAPP-A and BhCG were performed, with a sensitivity of $90 \%$ and a false positive rate of $3.2 \%$. 10 fetuses with trisomy 21 were observed. The sensitivity of nasal bone, ductus venosus and tricuspid regurgitation was $22.2 \%, 50 \%$ and $50 \%$ and specificity was $99.8 \%, 96.9 \%$ and $98.8 \%$ respectively. The overall sensitivity of contingent test was $90 \%$, with a reduction in false positive rate to $1.6 \%$, which would decrease the number of amniocentesis from 171 to 148 . Conclusion: The contingent test is a good tool to reduce the rate of false positives with respect to the combined test without decreasing the detection rate and thereby reduce the rate of invasive testing.

KEY WORDS: Trisomy 21, contingent test, secondary sonographic markers, nasal bone, ductus venosus, tricuspid regurgitation 


\section{INTRODUCCIÓN}

Durante el primer trimestre de gestación se realiza el screening poblacional con el test combinado para seleccionar las pacientes con alto riesgo de presentar un feto con síndrome de Down o Trisomía 21. El test combinado considera la edad materna, los marcadores bioquímicos séricos como la proteína plasmática A asociada al embarazo (PAPP-A) y la fracción $B$ libre de la hormona coriónica humana (BhCG) y los marcadores ecográficos como la traslucencia nucal. Este screening presenta una tasa de detección del $90 \%$ con una tasa de falsos positivos del $5 \%(1)$.

Con este test de cribado se estratifican a las pacientes en tres niveles de riesgo: a. bajo riesgo cuando la probabilidad de aneuploidía es $<1 / 1000$, b. riesgo intermedio entre $1 / 101$ y $1 / 1000$ y c. alto riesgo $\geq 1 / 100$ (1). El índice de riesgo a partir del cual se recomienda realizar una prueba invasiva se decide enfrentándolo con el riesgo de pérdida fetal tras la realización de ésta el cual es 1/200 (2). Por tanto, en nuestro hospital se ha consensuado que el límite para definir un alto riesgo y por tanto, ofrecer la realización de una prueba invasiva es $\geq 1 / 250$.

En diferentes estudios, se han investigado otros marcadores ecográficos de alteraciones cromosómicas y así mejorar la estrategia de cribado. Nicolaides y cols (1) en 2005, propusieron la onda a de flujo del ductus venoso, el hueso nasal y la regurgitación tricuspídea, los cuales hoy en día están establecidos como marcadores de segunda línea relacionados con cromosomopatías, estableciéndose a partir de ahí el llamado cribado contingente $(1,3,4)$. Con este tipo de cribado a las pacientes con riesgo intermedio en el test combinado, se les completa el estudio con estos marcadores secundarios, recalculándose el riesgo de trisomía 21.

El objetivo de este estudio es analizar los resultados de este cribado contingente estableciendo el punto de corte de alto riesgo en $\geq 1 / 100$ y determinar la efectividad de dichos marcadores ecográficos para discriminar, dentro de las pacientes con un riesgo intermedio las que son de menor riesgo, con el fin de evitar un excesivo número de pruebas invasivas y los efectos adversos y costes derivados de ellas.

\section{PACIENTES Y MÉTODOS}

Se calculó el riesgo de trisomía 21 y 18 mediante el test combinado en todas las gestantes entre la semana 11 y 13+6 de gestación que dieron el consentimiento para ello. Se extrajo sangre materna para la determinación de la PAPP-A y la BhCG y posteriormente se realizó la ecografía según los criterios de la Fetal Medicine Fundation (5) para medir la longitud cráneo-caudal (LCC) y la traslucencia nucal (TN). Los valores de la PAPP-A, BhCG y TN se convirtieron en múltiplos de la mediana (MoMs) para la edad gestacional correspondiente y se calculó el riesgo utilizándose para ello el programa informático PRISCA.

Tras esta primera etapa a aquellas pacientes con un riesgo entre 1/101 y 1/1000 se les realizó el sonograma genético, valorándose los marcadores ecográficos de segunda línea (la onda a del flujo del ductus venoso (DV) catalogándola como normal si era positiva o ausente o anormal si era reversa, el hueso nasal (HN) normal si estaba presente y anormal si estaba ausente y la regurgitación tricuspídea (RT) normal si era menor de $60 \mathrm{~mm} / \mathrm{seg}$ y duraba menos del $50 \%$ de la sístole o anormal si era mayor). Las ecografías se realizaron con un ecógrafo Voluson 750 Expert por cuatro ecografistas de la unidad de diagnóstico prenatal. Se calculó la sensibilidad y la tasa de falsos positivos mediante tablas de contingencia. Posteriormente, se recalcula el riesgo, si era $\geq 1 / 250$ se ofrecía la realización de una prueba invasiva y si el riesgo era menor se continuaba el control del embarazo normal.

Finalmente, los datos cromosómicos fetales se recogieron del cariotipo fetal si fue sometida a una prueba invasiva o de la valoración neonatal tras el nacimiento en caso contrario, reflejándose como sano aquel recién nacido no afecto de trisomía 21 y como síndrome de Down el que lo presentaba.

Los datos fueron recogidos en una base de datos y fueron analizados de manera retrospectiva utilizando el programa estadístico IBM SPSS Statistics® Versión 22.

\section{RESULTADOS}

Desde Enero de 2014 a Mayo de 2015 se realizaron 2.660 test combinados del primer trimestre valorándose la edad materna, la traslucencia nucal, la PAPP-A y la ßhCG. Resultando 93 de ellos con alto riesgo con un punto de corte $\geq 1 / 250$, 46 de ellas presentaban un riesgo mayor o igual a $1 / 100$, 218 con un riesgo intermedio (1/101-1/1000) y 2.395 con un riesgo bajo $(<1 / 1000)$.

La edad media de las pacientes fue de 32,1 años (rango: 15-50 años), la edad gestacional media fue de 12 semanas y un día y la LCC media fue de 63,4 mm (rango: 45-83 $\mathrm{mm}$ ).

En esas 2.660 gestaciones evaluadas hubo 10 fetos con trisomía 21: 9 tuvieron un alto riesgo $(\geq 1 / 250)$ en el test de cribado combinado y 1 bajo riesgo (<1/1000). De este modo, el test combinado que tiene en cuenta la edad materna, los marcadores bioquímicos y los marcadores ecográficos, estableciendo el punto de corte en $1 / 250$, tiene una sensibilidad de detección de trisomía 21 del $90 \%$ (9/10), una especificidad del 96,8\% (2.566/2.650), con una tasa de falsos positivos del 3,2\% (84/2.650) y por tanto, un valor predictivo negativo del $99,9 \%$ (2.566/2.567). 
Para realizar el análisis del test contingente se escogió un punto de corte de alto riesgo en $\geq 1 / 100$, encontrándose 46 pacientes con este riesgo en el test de screening, 7 en pacientes con fetos afectos de trisomía 21 y 39 con hijos normales, obteniendo una sensibilidad del $70 \%(7 / 10)$ y una tasa de falsos positivos del 1,5\% (39/2.650). Con este punto de corte, encontramos 218 pacientes con un riesgo intermedio entre 1/101-1/1000 a las que se reevaluó con los marcadores secundarios con el fin de obtener un nuevo riesgo individual. Entre ellas, presentaron 4 el DV anormal (1 feto con trisomía 21 y 3 normales), 1 alteración en el hueso nasal (en un feto normal) y 3 la regurgitación tricuspídea (1 feto con Síndrome de Down y un feto normal). Por tanto, el cribado contingente, denominando así a la combinación de un alto riesgo con el punto de corte de alto riesgo en $\geq 1 / 100$ en el test combinado, más la presencia de al menos un marcador ecográfico secundario alterado en pacientes con riesgo intermedio, presenta una sensibilidad del 90\% (9/10), con una reducción de la tasa de falsos positivos al $1,6 \%(44 / 2.650)$.
El hueso nasal (Tabla I) fue valorado en 2.600 pacientes: 2.591 fetos cromosómicamente normales y 9 con trisomía 21. El HN estaba ausente en el $22,2 \%(2 / 9)$ de los fetos con trisomía 21 y $0,2 \%$ $(5 / 2.590)$ de los fetos cromosómicamente normales, con una sensibilidad del $22,2 \%$ y una especificidad del $99,8 \%$.

El ductus venoso (Tabla II) fue valorado en 1.103 gestaciones: 1.097 eran fetos cromosómicamente normales y 6 con trisomía 21 . El DV fue anormal en el $50 \%(3 / 6)$ de los fetos con trisomía 21 y 3,1\% (34/1.097) de fetos cromosómicamente normales, con una sensibilidad del $50 \%$ y una especificidad del $96,9 \%$.

La regurgitación tricuspídea (Tabla III) fue valorada únicamente en 176 pacientes con riesgo intermedio entre 1/101-1/1000: 174 eran fetos normales y 2 con síndrome de Down, encontrándose 3 fetos con alteración en la regurgitación tricuspídea, 2 fetos normales y 1 síndrome de Down. Teniendo una sensibilidad del $50 \%$ y una especificidad del $98,8 \%$.

Tabla I

HUESO NASAL

\begin{tabular}{lllccc}
\hline & & & \multicolumn{2}{c}{ Hueso nasal } \\
& & & Presente & Ausente & Total \\
\hline RESULTADO & \multirow{2}{*}{ Normal } & Recuento & 2.586 & 5 & 2.591 \\
CARIOTIPO & & \% dentro de RESULTADO CARIOTIPO & $99,8 \%$ & $0,2 \%$ & $100,0 \%$ \\
\cline { 2 - 6 } & \multirow{2}{*}{ S. Down } & Recuento & 7 & 2 & 9 \\
& & \% dentro de RESULTADO CARIOTIPO & $77,8 \%$ & $22,2 \%$ & $100,0 \%$ \\
\hline \multirow{2}{*}{ Total } & Recuento & 2.593 & 7 & 2.600 \\
& & $\%$ dentro de RESULTADO CARIOTIPO & $99,7 \%$ & $0,3 \%$ & $100,0 \%$ \\
\hline
\end{tabular}

\section{Tabla II DUCTUS VENOSO}

\begin{tabular}{|c|c|c|c|c|c|}
\hline & & & \multicolumn{3}{|c|}{ Ductus Venenoso } \\
\hline & & & Normal & Patológico & Total \\
\hline \multirow{4}{*}{$\begin{array}{l}\text { RESULTADO } \\
\text { CARIOTIPO }\end{array}$} & Normal & Recuento & 1.063 & 34 & 1.097 \\
\hline & & \% dentro de RESULTADO CARIOTIPO & $96,9 \%$ & $3,1 \%$ & $100,0 \%$ \\
\hline & S. Down & Recuento & 3 & 3 & 6 \\
\hline & & \% dentro de RESULTADO CARIOTIPO & $50,0 \%$ & $50,0 \%$ & $100,0 \%$ \\
\hline \multirow[t]{2}{*}{ Total } & & Recuento & 1.066 & 37 & 1.103 \\
\hline & & \% dentro de RESULTADO CARIOTIPO & $96,6 \%$ & $3,4 \%$ & $100,0 \%$ \\
\hline
\end{tabular}




\section{Tabla III REGURGITACIÓN TRICUSPÍDEA}

\begin{tabular}{lllccc}
\hline & & & \multicolumn{3}{c}{ Regurgitación tricuspídea } \\
& & & Normal & Patológica & Total \\
\hline RESULTADO & \multirow{2}{*}{ Normal } & Recuento & 172 & 2 & 174 \\
& & \% dentro de RESULTADO CARIOTIPO & $98,9 \%$ & $1,1 \%$ & $100,0 \%$ \\
\cline { 2 - 6 } & S. Down & $\begin{array}{l}\text { Recuento } \\
\text { \% dentro de RESULTADO CARIOTIPO }\end{array}$ & 1 & 1 & 2 \\
\hline Total & & Recuento & 173 & $30,0 \%$ & $100,0 \%$ \\
\hline
\end{tabular}

En las pacientes con riesgo superior a 1/1000 la presencia de al menos un marcador ecográfico de anomalía cromosómica se observó en el $50 \%$ (5/10) de los fetos con trisomía 21 y en el $4,9 \%$ (13/264) de los fetos cromosómicamente normales, y la presencia de dos marcadores alterados en 1 feto con trisomía 21 y 1 normal.

En global, se realizaron 171 amniocentesis de las cuales 23 fueron realizadas en pacientes con un riesgo intermedio en el cribado, 74 en pacientes con alto riesgo y 74 con bajo riesgo. Se detectaron 9 fetos con trisomía 21, 1 trisomía $18(47 X Y+18)$, 1 trisomía $15(47 X Y+15)$, 1 trisomía $X(47 X X X)$, 3 monosomía $X(45 X 0), 1$ traslocación $(t(7 ; 22)$ (p13;q13)), 4 inversiones 2((invY p11.2q11.22); inv(9)(p11q13)) y 152 cariotipos normales. Las 23 pacientes con riesgo intermedio presentaron los marcadores secundarios normales por lo que su riesgo recalculado fue bajo. El resultado de las 23 amniocentesis fue en 22 un cariotipo normal y en 1 se observó una inversión $46 X X$,inv(9)(p11q13). Ésta inversión encontrada es un polimorfismo común en la población general, considerándose una variante de la normalidad.

\section{DISCUSIÓN}

En nuestro estudio la detección de pacientes con alto riesgo de trisomía 21 con el test combinado de primer trimestre presenta una sensibilidad del $90 \%$ con un tasa de falsos positivos del $3,2 \%$. Al añadir la detección de los marcadores ecográficos secundarios, se obtuvo una sensibilidad del $90 \%$ con una importante reducción de la tasa de falsos positivos al $1,6 \%$.

De las 171 amniocentesis realizadas en ese período de estudio, 23 fueron realizadas en pacientes con un riesgo intermedio que tras el test contingente pasaron a ser de bajo riesgo. Por tanto, se podrían haber evitado las 23 amniocentesis, no afectando a la detección de los casos de síndrome de Down y reduciendo las complicaciones y gastos que conlleva la realización de una prueba invasiva.

En cuanto al análisis de los marcadores secundarios por separado, presentan una alta especificidad, siendo la sensibilidad más baja que la obtenida en estudios previos.

En 2005, Nicolaides y cols (1), observaron que la ausencia o presencia de estos marcadores secundarios se daban con más frecuencias en fetos con trisomía 21 añadiéndolos como marcadores secundarios para la detección de estos fetos. Obtuvo una tasa de detección de trisomía 21 del 92\% para la ausencia de hueso nasal, con una tasa de falsos positivos del $2,1 \%$, del $94,2 \%$ para el aumento de la impedancia de la onda de ductus venoso con una tasa de falsos positivos del $2,7 \%$ y del $91,7 \%$ para la regurgitación tricuspídea con una tasa de falsos positivos del $2,7 \%$.

El estudio FASTER (6) compara tres métodos de detección de pacientes con alto riesgo de cromosomopatía, el cribado secuencial, integrado y contingente, siendo las tasas de detección y de falsos positivos similares entre ellos con datos ligeramente peores para el contingente, sin embargo, el cribado contingente disminuye el número de pacientes que tiene que volver en el segundo trimestre para completar el estudio reduciendo el número de controles por paciente.

En posteriores estudios se ha refutado la utilidad de esta estrategia de cribado, Ghaffari y cols (7) obtuvieron una reducción en la tasa de falsos positivos del $4,8 \%$ al 3,7\% para la detección de fetos con trisomía 21 al añadir la valoración de estos marcadores secundarios. Posteriormente MuñozCortes y cols (4) en 2012, realizaron un estudio multicéntrico en el que obtuvieron una reducción de la tasa de falsos positivos del $3 \%$ al $1,8 \%$ sin disminuir la tasa de detección.

Los datos de nuestro estudio deben interpretarse con cautela debido al pequeño tamaño de muestra del estudio, por lo que las tasas de sensibilidad 
de los marcadores son relativamente más bajas que en otros estudios, sin embargo, los datos del test contingente reflejan datos similares a los estudios previos con una gran reducción de la tasa de falsos positivos y descenso en el número de pruebas invasivas en pacientes con riesgo intermedio.

\section{CONCLUSIÓN}

El cribado contingente es una buena estrategia de cribado para pacientes con riesgo intermedio en el test combinado, ya que presenta una tasa de detección similar y reduce de manera importante la tasa de falsos positivos. Por tanto, supone una buena herramienta para disminuir el número de pruebas invasivas innecesarias sin disminuir la detección de fetos con trisomía 21.

\section{REFERENCIAS}

1. Nicolaides KH, Spencer K, Avgidou K, Faiola S, Falcon O. Multicenter study of first-trimester screening for trisomy 21 in 75821 pregnancies: Results and estimation of the potential impact of individual risk-oriented two-stage first-trimester screening. Ultrasound Obstet Gynecol. 2005;25(3):221-6.

2. Consenso Ecografía 2009 [Internet]. Disponible en: http://www.prosego.com/categoria-consenso/ecografia/
3. Gómez A, Prieto MA, Cid MJ, López MJ, Ávila S, Repollés M. Cribado contingente para la detección de trisomía 21 en el primer trimestre de la gestación mediante marcadores ecográficos de segunda línea. Prog Obstet Ginecol. 2013;56(3):129-34.

4. Muñoz-Cortes $M$, Arigita $M$, Falguera $G$, Seres $A$, Guix D, Baldrich $E$, et al. Contingent screening for Down syndrome completed in the first trimester: A multicenter study. Ultrasound Obstet Gynecol. 2012;39(4):396-400.

5. Nicolaides $\mathrm{KH}$, Falcón $\mathrm{O}$. The 11-13+6 weeks scan. Fetal Med Found [Internet]. 2004. Disponible en: http://www.fetalmedicine.com/synced/fmf/FMF-spanish.pdf

6. Cuckle HS, Malone FD, Wright D, Porter TF, Nyberg $\mathrm{DA}$, Comstock $\mathrm{CH}$, et al. Contingent screening for Down Syndrom-results from de FaSTER trial. Prenat Diagn. 2008;28(2):89-94.

7. Ghaffari SR, Tahmasebpour AR, Jamal A, Hantoushzadeh S, Eslamian L, Marsoosi V, et al. First-trimester screening for chromosomal abnormalities by integrated application of nuchal translucency, nasal bone, tricuspid regurgitation and ductus venosus flow combined with maternal serum free B-hCG and PAPP-A: A 5 -year prospective study. Ultrasound Obstet Gynecol. 2012;39(5):528-34. 